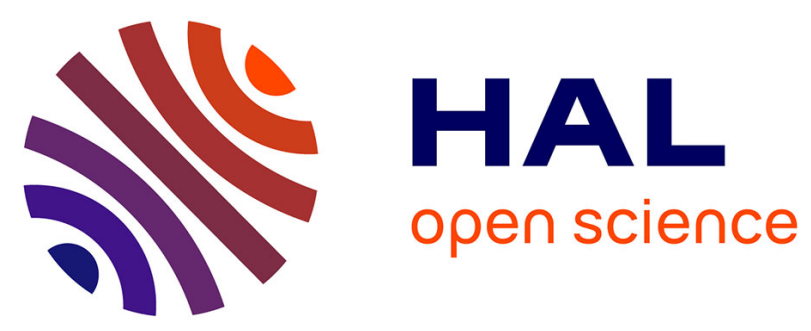

\title{
Réalisation de microponts supraconducteurs de niobium par bombardement ionique
}

\author{
R. Adde, P. Crozat, S. Gourrier, G. Vernet, M. Bernheim, D. Zenatti
}

\section{To cite this version:}

R. Adde, P. Crozat, S. Gourrier, G. Vernet, M. Bernheim, et al.. Réalisation de microponts supraconducteurs de niobium par bombardement ionique. Revue de Physique Appliquée, 1973, 8 (4), pp.455-461. 10.1051/rphysap:0197300804045500 . jpa-00243714

\section{HAL Id: jpa-00243714 https://hal.science/jpa-00243714}

Submitted on 1 Jan 1973

HAL is a multi-disciplinary open access archive for the deposit and dissemination of scientific research documents, whether they are published or not. The documents may come from teaching and research institutions in France or abroad, or from public or private research centers.
L'archive ouverte pluridisciplinaire HAL, est destinée au dépôt et à la diffusion de documents scientifiques de niveau recherche, publiés ou non, émanant des établissements d'enseignement et de recherche français ou étrangers, des laboratoires publics ou privés. 


\title{
RÉALISATION DE MICROPONTS SUPRACONDUCTEURS DE NIOBIUM PAR BOMBARDEMENT IONIQUE (*)
}

\author{
R. ADDE, P. CROZAT, S. GOURRIER, G. VERNET
}

Institut d'Electronique Fondamentale, Laboratoire associé au CNRS, Bâtiment 220, Université Paris 11, 91405 Orsay, France

\section{BERNHEIM}

Laboratoire de Physique des Solides, Bâtiment 510, Laboratoire associé au CNRS, Université Paris 11, 91405 Orsay, France

\section{ZENATTI}

LETI-DINR, Centre d'Etudes Nucléaires, Cedex 85, 38041 Grenoble, France

\begin{abstract}
Résumé. - On présente une technique reproductible de fabrication de microponts supraconducteurs applicable aux supraconducteurs « durs » $(\mathrm{Ta}, \mathrm{V}, \mathrm{Nb})$ par bombardement ionique. L'usinage de microponts est obtenu globalement en formant sur un film mince une image ionique représentant la structure à usiner. Les propriétés de microponts de niobium ainsi réalisés sont ensuite décrites : température critique, résistance normale, caractéristique $(I, V)$, action d'un champ magnétique continu et d'un champ hyperfréquence.
\end{abstract}

Abstract. - Superconducting microbridges of « hard » superconductors can be realized reproducibly using an ion bombardment technique. The micromachining is obtained directly if one focusses onto the sample an ionic image which represents the structure to be machined. Niobium microbridges have been realized using this technique and their properties are described : critical temperature, normal resistance, $I-V$ characteristic, $d-c$ magnetic field effect and microwave field effect.

On désigne par le terme général de «jonction Josephson » tous les dispositifs supraconducteurs à couplage faible, quel que soit le mécanisme détaillé de transport du supracourant dans le système. Ceci vient de leur caractéristique commune qui est de vérifier au moins en première approximation, les relations déterminées par Josephson pour les jonctions tunnels supraconductrices [1]. Les dispositifs qui ont fait l'objet des études les plus détaillées pendant la dernière décade sont d'une part les jonctions tunnels supraconductrices [2] et d'autre part les contacts supraconducteurs à pointe [3]. Les travaux sur les microponts n'ont pas été poursuivis avec la même vigueur. Les microponts sont constitués par un film mince supraconducteur présentant dans sa partie médiane une région de petites dimensions ayant une supraconductivité affaiblie : ils ont donc la caractéristique essentielle de former un circuit entièrement supraconducteur. La fabrication de films minces supraconducteurs (épaisseur de quelques centaines d'Angströms) de bonne qualité relève d'une techno-

(*) Cette recherche fait l'objet d'un contrat de la DRME. logie qui est maintenant bien dominée. Par contre la réalisation de régions inhomogènes (microponts) présente de sérieuses difficultés parce qu'elles doivent avoir de très petites dimensions $(\sim 0,5 \times 0,5 \mu)$. Les difficultés rencontrées ont conduit, après les travaux de Dayem et Wiegand en 1967 [4] à un certain ralentissement des publications dans ce domaine.

Les caractéristiques que devraient avoir idéalement de tels dispositifs sont essentiellement :

a) Une impédance normale (lorsque $I \gg I_{\mathrm{c}}$ ou $T>T_{\mathrm{c}}$ ) de l'ordre d'au moins quelques ohms. Si une jonction est utilisée pour la détection de rayonnement hyperfréquence, on peut réaliser des guides d'ondes amincis dont l'impédance caractéristique soit dans la gamme 1-10 $\Omega$. Afin d'obtenir une jonction ayant une impédance de cet ordre, il faut non seulement que le micropont ait une faible conductivité, mais aussi que la capacité shunt $C$ du dispositif soit faible.

b) Un courant critique $I_{c}$ compris entre $10^{-5}$ et $10^{-7} \mathrm{~A}$ suivant les applications. Il est très important, pour les applications à tension finie, que la caractéristique $(I, V)$ ne présente pas d'hystérésis dans la zone. 
opérationnelle. Ceci implique comme l'a souligné McCumber [5] une constante de temps normale de la jonction $R_{\mathrm{N}} C$ petite par rapport à la fréquence de plasma Josephson $\left(2 I_{\mathrm{c}} / h C\right)^{1 / 2}$. L'hystérésis de la caractéristique $(I, V)$ peut d'autre part provenir d'effets thermiques si la conductivité thermique entre le micropont et le bain est trop faible, ou si le courant critique est trop sensible aux variations de température.

c) Une gamme de température de fonctionnement aussi importante que possible au-dessous de la température critique du pont mais largement supérieure à $0,5 \mathrm{~K}$. Il est souhaitable que le reste du film supraconducteur menant au micropont ait une température critique suffisamment inférieure à celle du pont. Si cela n'est pas le cas des fluctuations de point critique de la phase de la fonction d'onde le long du parcours supraconducteur peuvent produire une dégradation des effets d'interférence quantique. Près de $T_{\mathrm{c}}$ également des déplacements de lignes de vortex à travers le film peuvent produire des glissements de phase multiples de $2 \pi$ et une dissipation résistive associée. Un dispositif dont le domaine de température de fonctionnement comprend la température d'ébullition de l'hélium liquide sous pression normale $(4,2 \mathrm{~K})$ présente de plus un intérêt pratique considérable.

d) Une certaine insensibilité aux champs magnétiques continus extérieurs pour éviter les blindages magnétiques encombrants et onéreux.

e) Une aussi bonne résistance que possible aux cycles thermiques répétés et à l'exposition à l'air et à l'humidité.

Les principales réalisations à l'heure actuelle dans le domaine des microponts supraconducteurs sont :

- Les ponts de Dayem étudiés par Gregers-Hansen et al. [6], Yeong-du-Song et G. I. Rochlin [7] où la constriction est réalisée par gravure mécanique dans un supraconducteur "mou » $(\mathrm{Sn})$. Des constrictions de très petites dimensions ont été réalisées $(\sim 0,2 \times 0,5 \mu)$ qui assurent des propriétés hyperfréquences intéressantes. Mais ces ponts doivent être opérés à une température inférieure à la température critique de l'étain $(3,8 \mathrm{~K})$, et voisine de celle-ci (quelques centièmes de $\mathrm{K}$ ) étant donné l'augmentation très rapide du courant critique lorsque la température décroît $(2 \mu \mathrm{A}$ par $\mathrm{mK})$. Leur courant critique est d'autre part très sensible à un champ magnétique perpendiculaire à la surface du film.

- Les ponts de Notarys [8]-[10] où un dépôt de métal normal dans la zone du micropont affaiblit localement par effet de proximité la supraconductivité. On doit utiliser des couples de métaux normalsupraconducteur ne diffusant pas de façon trop importante tels que $\mathrm{Sn}-\mathrm{Au}, \mathrm{Pb}-\mathrm{Cu}$, mais les films de plomb résistent mal à une exposition prolongée à l'air et l'humidité. Ces ponts sont également très sensibles au champ magnétique.
- Les microponts en supraconducteurs durs ( $\mathrm{Nb}$, Ta) où l'affaiblissement de la supraconductivité du micropont est effectué en amincissant le film dans cette zone par oxydation anodique [8]. Pour obtenir des courants critiques convenables le film principal doit être très mince (100-500 $\AA$ ) et dans ce cas la température critique des films de Ta tombe en-dessous de 4,2 K ; d'autre part les films de Ta sont assez sensibles à l'oxydation de l'air. Les ponts réalisés à partir de films en niobium possèdent d'excellentes propriétés ; il est assez difficile de réaliser des constrictions de très petites dimensions.

- Récemment enfin, des microponts présentant des constrictions de petites dimensions ont été réalisés par photogravure oùle motif est impressionné avec une définition élevée sur une résine sensible aux électrons avec un microscope électronique à balayage. Le métal (In ou Sn) est attaqué soit chimiquement soit par pulvérisation cathodique (F. Auracher et S. Mc Carthy, communications privées).

Nous avons mis au point et présentons ici une nouvelle technique de réalisation de microponts à partir de films de supraconducteur «dur» (niobium) qui ont à la fois une constriction de petites dimensions $(\sim 0,6 \times 0,6 \mu)$ ce qui est indispensable pour leur bon comportement hyperfréquence et un affaiblissement suffisant de la supraconductivité dans cette zone pour qu'ils opèrent à 4,2 K. Leurs autres avantages sont de fonctionner dans une gamme de température importante $(>2 \mathrm{~K})$ et d'être peu sensibles à un champ magnétique continu. Cette technique utilise la méthode d'usinage par bombardement ionique étudiée par M. Bernheim et R. Castaing [10], [11], [12]. Nous décrivons dans la première partie de cet article la technique de fabrication des microponts. Dans la deuxième partie nous donnons les premiers résultats obtenus dans l'étude de leurs propriétés.

1. Technique de fabrication des microponts. - Des ébauches de microponts (Fig. 1) sont réalisées sur des films minces de niobium, puis une constriction $(\sim 0,6 \times 0,6 \mu)$ est usinée par bombardement ionique.

1.1 RÉALISATION DES ÉBAUCHES. - 1.1.1 Films de niobium. - Un film mince (400 à $600 \AA$ ) de niobium supraconducteur est déposé sur un substrat circulaire $(\varnothing=20 \mathrm{~mm})$ de silicium. Le choix de ce type de substrat est imposé par la méthode d'usinage ionique qui s'effectue à température ambiante, pour permettre l'écoulement des charges électriques et éviter les décharges disruptives particulièrement en fin d'opération. A 4,2 K, ces substrats sont isolants. L'évaporation du niobium est effectuée avec un canon à électrons car c'est un matériau réfractaire dont la température d'évaporation est très élevée. Il ne peut être obtenu en films minces supraconducteurs que lorsqu'il est déposé sur des substrats chauffés à température élevée $\left(400^{\circ} \mathrm{C}\right)$ dans un vide poussé et propre exempt d'hydrocarbures 


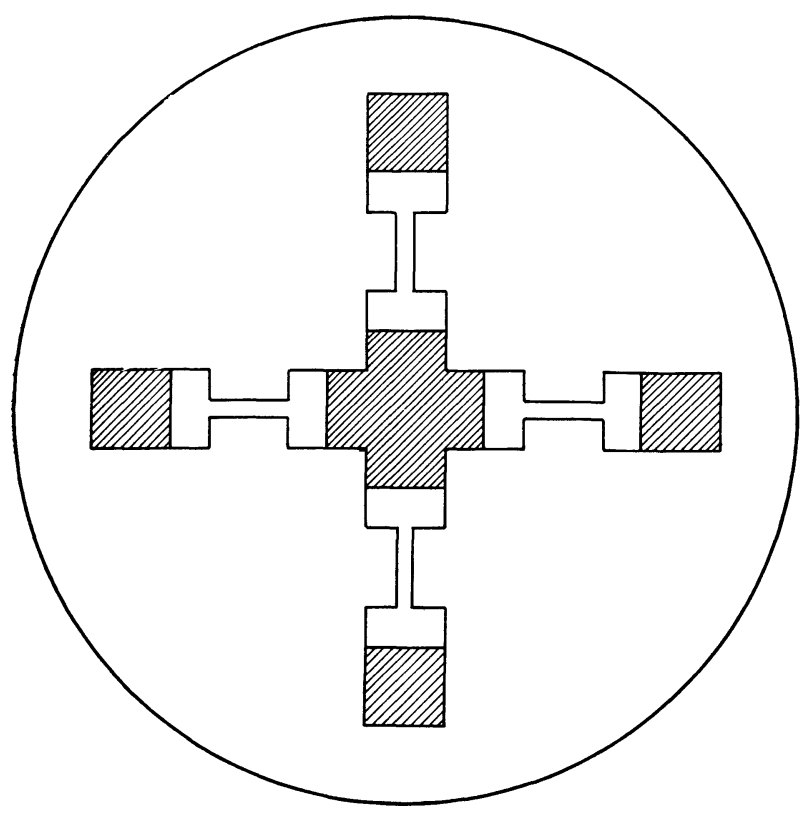

Fig. 1. - Motif servant à l'usinage des microponts ; le substrat est une pastille de silicium ( $\varnothing=20 \mathrm{~mm}$, épaisseur $0,2 \mathrm{~mm}$ ), l'épaisseur du film de niobium est $600 \AA$. Avec ce motif, on usine un micropont $(\sim 0,6 \times 0,6 \mu)$ au milieu de chaque canal de largeur $250 \mu$. Dans l'appareil d'usinage ionique, un support tournant excentré permet d'effectuer successivement l'usinage de chaque pont. La pastille de Si est découpée après l'usinage et les connexions électriques (fils d'Au) sont fixées sur les plots de contact en $\mathrm{Au}$ par thermocompression ou avec de la laque d'argent.

et bien entendu d'oxygène, le niobium étant un excellent getter. Le cycle d'évaporation se résume ainsi :

a) Après avoir placé les substrats nettoyés sur le porte-substrat chauffant, on effectue la descente en vide à l'aide de pompes à cryosorption pour le vide primaire et d'une pompe ionique avec sublimateur de titane pour le vide secondaire.

b) Le dégazage des substrats par chauffage est déclenché lorsque l'on atteint un vide de l'ordre de $10^{-7}$ torr et se poursuit pendant 10 à $12 \mathrm{~h}$.

c) Dans un même temps, les substrats étant protégés par un cache, on effectue des cycles réguliers d'évaporation qui assurent le dégazage du lingot de niobium. Le niobium ainsi évaporé, excellent getter, va recouvrir les parois de l'enceinte améliorant ainsi le vide limite $\left(\sim 10^{-8}\right.$ torr $)$.

d) L'évaporation du niobium purifié restant a alors lieu sur les substrats. Elle est pilotée par un oscillateur à quartz. A ce stade le vide est généralement inférieur à $10^{-7}$ torr.

e) Les substrats se refroidissent lentement. Lorsqu'ils atteignent $120^{\circ} \mathrm{C}$ environ, on évapore une mince couche d'or qui servira aux prises de contact sur les ponts réalisés. L'enceinte à vide n'est ouverte que lorsque la température des substrats est inférieure à $60^{\circ} \mathrm{C}$.

Ces conditions ont permis de réaliser des films de
$\mathrm{Nb}$ d'épaisseur $600 \AA$ ayant une température critique de $8 \mathrm{~K}$ et des rapports de résistivité

$$
\rho=R_{300 \mathrm{~K}} / R_{77 \mathrm{~K}} \sim 2,8 .
$$

Ce rapport de résistivités particulièrement élevé est un critère de l'excellente qualité des films.

1.1.2 Photomasquage et gravure chimique. - Sur le film déposé sur chaque substrat, on réalise les ébauches de ponts constituées par un canal rectangulaire étroit $(250 \mu)$ suivant le motif de la figure 1. La taille du canal est imposée par le champ opératoire de l'appareil d'usinage ionique. Au moyen d'un premier photomasquage, la résine après insolation et développement a une géométrie semblable à celle de la figure 1 . Pour protéger le motif, on procède alors à la gravure de l'or qui est immédiatement suivie par celle du niobium. Les plots de contact en or sont obtenus après un deuxième photomasquage qui élimine l'or du reste du motif.

\subsection{USINAGE D'UN MICROPONT PAR BOMBARDEMENT} IONIQUe [10], [12]. - On remplace les techniques de photogravure optique par un procédé d'usinage utilisant un dispositif d'optique ionique. L'usinage par pulvérisation cathodique que produit un bombardement ionique ne nécessite pas de fortes densités puisque chaque ion pulvérise une quantité donnée de matériau indépendamment des autres ions du faisceau. La finesse de l'usinage est indépendante théoriquement de la densité ionique qui tombe sur l'échantillon; la pulvérisation d'une région donnée ne dépend que du nombre total d'ions qui l'ont bombardée ainsi que de leur nature et de leur énergie.

L'usinage par bombardement ionique est obtenu globalement sans balayage mécanique ou électrique, en formant sur la cible une image ionique qui représente la structure à usiner. L'appareil de micro-usinage (Fig. 2) est donc constitué principalement par une optique ionique réductrice qui reproduit sur la cible l'ombre du modèle; on usine dans l'échantillon des ouvertures qui correspondent aux parties transparentes du modèle pour obtenir ainsi une réplique positive en miniature de celui-ci.

Le champ usinable de l'appareil étant $\sim \varnothing=600 \mu$, le canal de $250 \mu$ du motif (Fig. 1) est facilement centré, Le facteur de réduction de l'optique ionique étant 17 , pour obtenir un micropont de l'ordre de 0,6 $\times 0,6 \mu$, on utilise le modèle de la figure 3 réalisé par deux lames métalliques minces distances de $10 \mu$ sur lesquelles est croisé un fil de tungstène $(\varnothing=10 \mu)$. Le rapport densité ionique au niveau de la cible/densité ionique au niveau du modèle est égal au carré du facteur de réduction soit environ 300 . Le taux de pulvérisation du modèle est donc très inférieur à celui de la cible ce qui assure au modèle une bonne longévité.

La finesse de l'usinage est obtenue par le choix d'une optique ionique soignée, c'est-à-dire une optique 
ELECTRON OPTIC

ION OPTIC
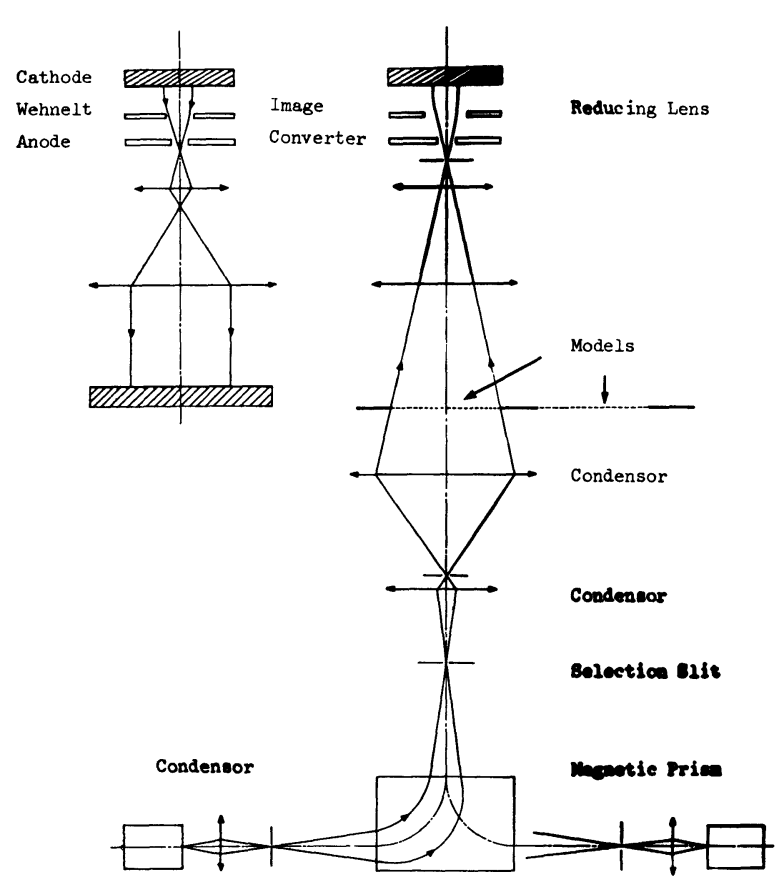

Ion Source 1

Ion Source 2

Fig. 2. - Diagramme optique de l'appareil de micro-usinage ionique. Les optiques ionique et électronique sont superposées dans la réalité. Les sources d'ions sont constituées par un arc basse tension avec un champ magnétique axial. La source d'ions $\mathrm{A}^{+}$produit un faisceau dont l'intensité après filtrage magnétique à $90^{\circ}$ est de l'ordre de $0,3 \mu \mathrm{A}$, correspondant à des densités à $0,4 \mu \mathrm{A} / \mathrm{mm}^{2}$ à la surface de la cible. Un condenseur électrostatique à trois électrodes éclaire le modèle en formant l'image du " cross over » de la source d'ions sur la pupille d'entrée du convertisseur ions-électrons ; il dirige ainsi vers la cible tous les ions qui ont traversé le modèle.

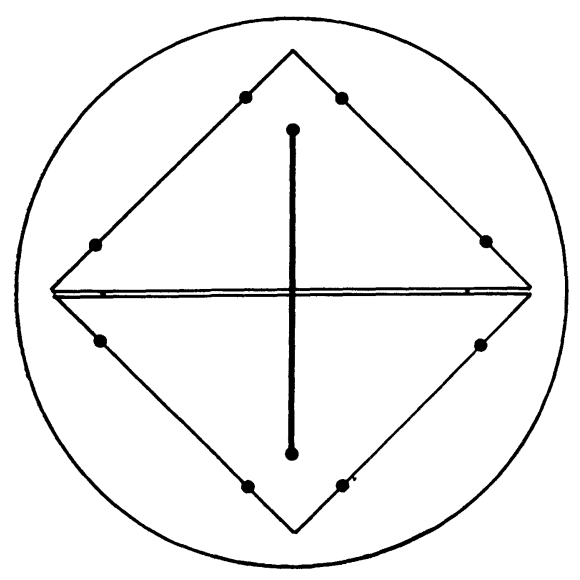

FIG. 3. - Modèle utilisé pour l'usinage par bombardement ionique des microponts. Il est constitué par une fente de largeur $10 \mu$ entre deux lames minces métalliques et d'un fil de tungstène $(\varnothing=10 \mu)$ croisé. On usine dans chaque canal d'un motif une ouverture correspondant aux parties transparentes du modèle ; après l'opération les deux côtés du canal ne sont plus reliés que par un micropont de supraconductivité affaiblie. comportant des faisceaux de faible ouverture et par conséquent de faible densité. Pour une optique donnée la finesse est limitée :

a) Par la qualité de l'image ionique projetée sur la cible liée aux aberrations de l'optique réductrice et à la précision de la mise au point. L'appareil de microusinage comporte un dispositif de contrôle de cette mise au point par une image électronique auxiliaire : on utilise l'émission secondaire d'électrons produite par le bombardement ionique. Ces électrons après accélération sont focalisés sur un écran fluorescent et on observe sur l'écran une image électronique qui constitue un agrandissement de l'image ionique au niveau de la cible.

b) Par les inégalités de rendement de pulvérisation de la couche polycristalline. La figure 4 montre un exemple de finesse d'usinage qui peut être obtenu avec cet appareil en utilisant comme modèle une grille.

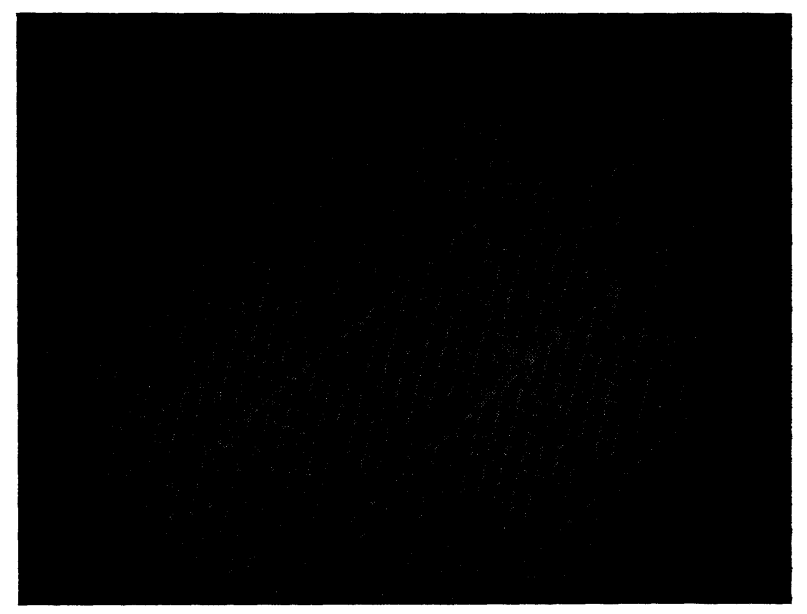

(a)

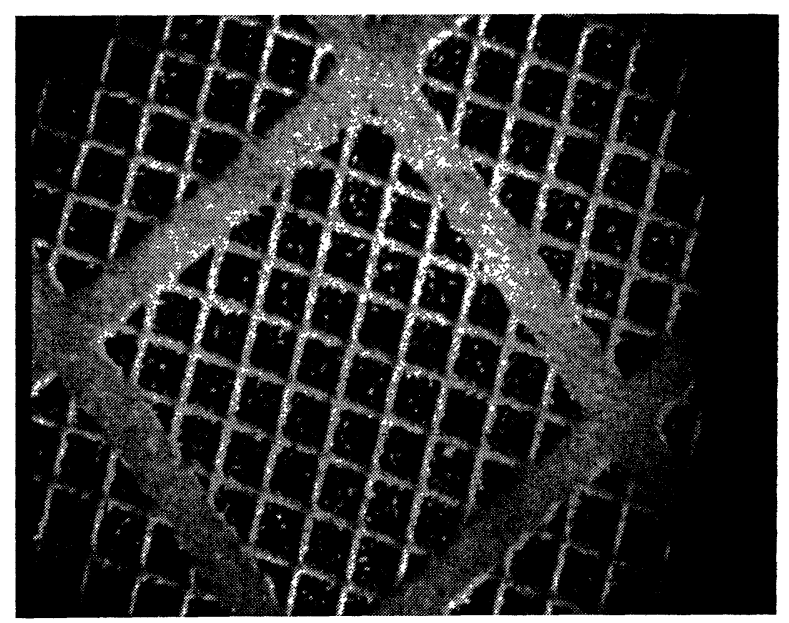

(b)

FIg. 4. - Observation au microscope électronique à balayage de grilles superposées de 1 et $6 \mu$ de pas, obtenues à partir de modèles de 18,5 et $100 \mu$ de pas. La distorsion que l'on peut observer provient de l'inclinaison de l'échantillon dans le microscope à balayage pour accroître le contraste de ces grilles ; elle n'est pas présente sur les cibles usinées par les ions. 
Le paramètre important caractérisant l'efficacité de l'usinage est le taux de pulvérisation cathodique $S$, rapport du nombre d'atomes arrachés au nombre d'ions incidents. Nous effectuons l'usinage de la cible de niobium avec un faisceau d'ions argon post-accélérés à $90 \mathrm{keV}$. Dans ce cas $S \sim 1$ [16]. La densité du faisceau ionique sur la cible est typiquement $0,4 \mu \mathrm{A} /$ $\mathrm{mm}^{2}$. Un calcul approché mené dans le cas idéal où l'on suppose un usinage parfaitement homogène et une densité atomique de la couche de niobium égale à celle $\mathrm{du}$ niobium monocristallin conduit à une durée d'usinage de $15 \mathrm{~min}$ pour une couche de $\mathrm{Nb}$ d'épaisseur $600 \AA$. Expérimentalement on trouve que les durées d'usinage sont supérieures (de l'ordre de $50 \%$ ), et que la précision est supérieure à $0,5 \mu$ (limite de précision du microscope optique). Une analyse approfondie des microponts au microscope électronique à balayage s'avère nécessaire, pour apprécier en particulier l'homogénéité du bombardement d'une couche polycristalline dont les gains peuvent atteindre quelques dizaines de microns.

2. Propriétés des microponts supraconducteurs de niobium. - 2.1 TeMPÉRATURE CRITIQUE. - Les microponts sont testés avec le dispositif de la figure 5 dont la température peut être régulée entre 1,5 et $9 \mathrm{~K}$.

Partant d'un film de niobium de $600 \AA$ ayant un $T_{\mathrm{c}}=8 \mathrm{~K}$, on constate que le bombardement ionique par un faisceau d'ions $\mathrm{Ag}^{+}$de forte énergie $(90 \mathrm{keV})$ diminue rapidement cette température critique. Dans toutes les expériences la densité du faisceau ionique est $0,4 \mu \mathrm{A} / \mathrm{mm}^{2}$. En utilisant pour modèle une fente complète, largeur $10 \mu$, au lieu du modèle de la figure 2 , on trouve qu'après une durée de bombardement de $t=6 \mathrm{~min}, T_{\mathrm{c}}=3,5 \mathrm{~K}$. La diminution de $T_{\mathrm{c}}$ est beaucoup plus lente lorsque l'on usine des ponts; avec le modèle de la figure 2 , pour

$t=6 \min \rightarrow T_{\mathrm{c}}=7 \mathrm{~K} ; t=16 \min \rightarrow T_{\mathrm{c}}=6,2 \mathrm{~K}$.

Théoriquement, comme les ponts réalisés sont courts, la température critique du pont devrait rester pratiquement égale à celle du film $(\sim 8 \mathrm{~K})$. En raison des aberrations de l'optique ionique et des défauts de réglage, et des dimensions très faibles du pont, celui-ci subit le bombardement d'une quantité non nulle d'ions $\mathrm{A}^{+}$ce qui explique cette diminution progressive de la température critique. Cet effet, qui est $a$ priori un défaut, est utilisé ici comme un avantage : il permet d'affaiblir la supraconductivité du micropont nettement au-dessous de celle du film principal (confère les qualités d'un micropont "idéal »). Des $T_{\mathrm{c}}$ compris entre 4,2 et $4,5 \mathrm{~K}$ ont ainsi été obtenus avec des films de $\mathrm{Nb}$ d'épaisseur $400 \AA$, correspondant à des courants critiques dans la gamme 20 à $100 \mu \mathrm{A}$ à 4,2 $\mathrm{K}$, mais au cours d'usinages de moins bonne qualité qui ont fourni des microponts de propriétés hyperfréquences un peu inférieures. Nous pouvons cependant déjà affirmer que la technique d'usinage par bombardement

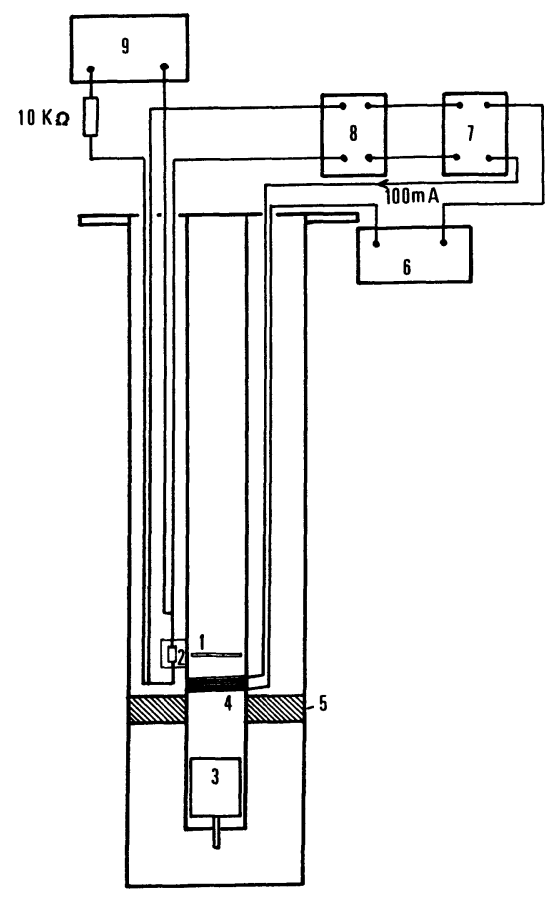

FIG. 5. - Tête du cryostat servant à tester les microponts entre 4,2 et $9 \mathrm{~K}: 1$ ) Micropont sous atmosphère d'hélium gazeux à pression réduite ; 2) Mesure de la température ; 3) Piston de court-circuit du guide hyperfréquence ; 4) Enroulement de chauffage ; 5) Contact thermique avec le bain d'hélium liquide extérieur à l'enceinte contenant le guide hyperfréquence ; 6) Alimentation continue de l'enroulement de chauffage; 8) Voltmètre numérique effectuant la mesure de température délivrant à la sortie une tension d'erreur amplifiée par l'amplificateur continu de puissance 7. Cet amplificateur est monté en série avec l'alimentation continue 6 et assure une stabilisation de température de l'ordre de $10^{-3} \mathrm{~K}$; 9) Alimentation continue de la sonde de température. Entre $1,5 \mathrm{~K}$ et $4,2 \mathrm{~K}$, l'enceinte 5 est retirée et la stabilisation de température est effectuée à partir de celle de la pression d'hélium avec un manostat cartésien.

ionique permet d'obtenir des dispositifs qui fonctionnent directement à la température de l'hélium liquide (cf. Fig. 7 et 8 ), et qu'il est important de pouvoir contrôler la «pollution » du pont le plus exactement possible.

2.2 RÉSISTANCE NORMALE $R_{\mathrm{N}}$ - - Les résistances sont toujours très faibles (quelques dixièmes d'ohm). Si l'on porte pour différents usinages $R_{\mathrm{N}}$ en fonction $\mathrm{du}$ produit courant ionique $\times$ temps de bombardement, on obtient un nuage de points sensiblement alignés. La fente transite à une température inférieure à celle du pont et celui-ci se trouve " shunté " par une résistance de quelques dixièmes d'ohm mesurée à partir de l'usinage de fentes complètes : en effet dans les expériences faites jusqu'à maintenant, l'usinage n'est pas total, c'est-à-dire qu'il reste toujours un peu de niobium de $T_{\mathrm{c}}$ très faible dans le canal. Il serait intéressant d'effectuer des usinages complets, sans toutefois trop diminuer le $T_{\mathrm{c}}$ du micropont, pour réaliser des dispositifs dont la résistance normale soit dans la gamme souhaitable (1-10 $\Omega$ ). 
2.3 Courant CRitique $I_{\mathrm{c}}$. - Pour les ponts au voisinage de $T_{\mathrm{c}}, I_{\mathrm{c}}=f(T)$ augmente d'abord assez lentement, puis ensuite varie linéairement sur une zone d'environ $2 \mathrm{~K}$ (Fig. 6). Lorsque la supraconductivité $\mathrm{du}$ micropont est suffisamment affaiblie, on obtient

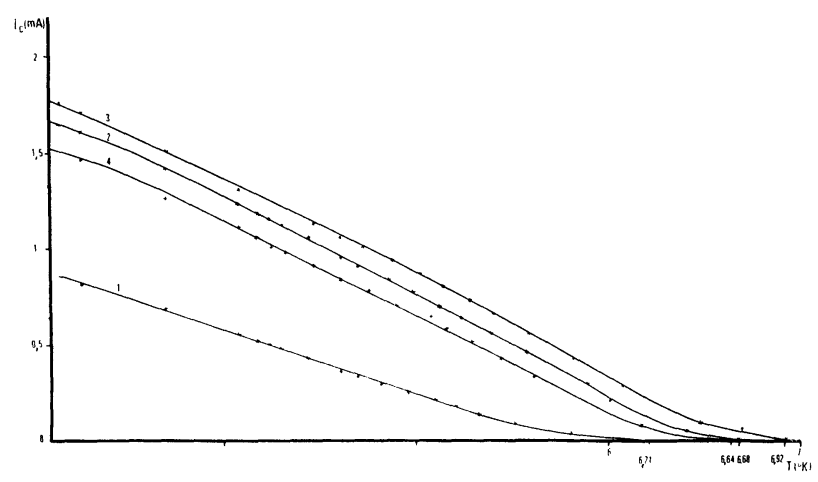

Fig. 6. - Variation du courant critique de microponts de niobium en fonction de la température pour plusieurs durées de l'usinage ionique ; 1) $16 \mathrm{~min}$; 2) $12 \mathrm{~min}$; 3) $10 \mathrm{~min}$; l'usinage $\mathrm{n}^{\circ} 4$ est inhomogène. La densité de courant sur la cible est $0,4 \mu \mathrm{A} / \mathrm{mm}^{2}$. L'influence d'un champ radiofréquence sur la caractéristique $I-V$ du pont no 2 est étudiée figures 7 et 8 .

des variations du courant critique en fonction de la température de $0,3 \mu \mathrm{A} / \mathrm{mK}$ et au-dessous, donc très inférieures à celles des microponts [6], [7] réalisés par gravure mécanique $(2 \mu \mathrm{A} / \mathrm{mK})$. Ceux-ci présentent une variation linéaire du courant critique au voisinage de $T_{\mathrm{c}}$ pour des ponts en Sn très courts (longueur $l$ de 0,2 à $0,5 \mu$ ). Dans ce cas la variation linéaire peut être expliquée à partir des résultats de Baratoff, Blackburn et Schwartz [14] qui ont résolu les équations de Ginzburg-Landau indépendantes du temps pour un modèle de micropont à une dimension : la longueur des microponts est alors inférieure à la longueur de cohérence à la température de fonctionnement. Cette condition est loin d'être remplie pour les ponts en $\mathrm{Nb}$ que nous avons expérimentés, et nous n'avons pas encore d'explication de la variation $I_{\mathrm{c}}=f(T)$ observée.

Rieger, Scalapino et Mercereau [15] ont récemment traité de façon théorique le processus de relaxation périodique appelé « glissement de phase » qui explique les propriétés de type Josephson des circuits entièrement supraconducteurs que sont les microponts. Ils ont pour cela résolu numériquement les équations de Ginzburg-Landau dépendant du temps en traitant un modèle à une dimension et prédisent une caractéristique $(I, V)$ où la caractéristique $(I-V)$ reste parallèle à forte tension à la droite $V / R_{\mathrm{N}}$ mais en est écartée de $\frac{1}{2} I_{\mathrm{c}}$. Ce résultat est confirmé par leurs expériences [8]-[10], et dans certains cas par les nôtres. Les écarts que nous observons sont probablement dus à la résistance normale du canal incomplètement usiné qui shunte le micropont.

2.4 RÉPONSE DES MICROPONTS A UN"RAYONNEMENT HYPERFRÉQUENCE. - La figure 7 montre l'influence

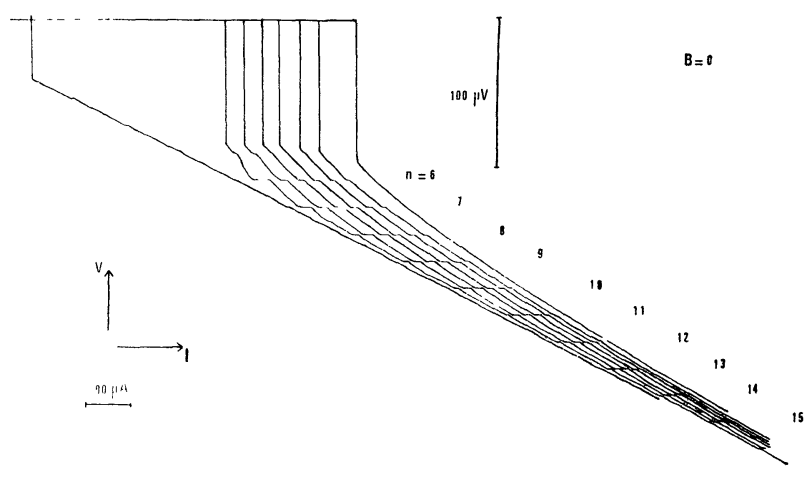

FIG. 7. - Caractéristique $I-V$ à $4,2 \mathrm{~K}$ en champ magnétique nul en fonction de la puissance hyperfréquence incidente $(\omega / 2 \pi \sim 9000 \mathrm{MHz})$ d'un micropont de niobium à supraconductivité faible. La température critique du film principal est $8 \mathrm{~K}$ et celle du micropont $6,68 \mathrm{~K}$.

d'un rayonnement hyperfréquence à $\sim 9000 \mathrm{MHz}$ mesurée à $4,2 \mathrm{~K}$ pour un micropont de $T_{\mathrm{c}}=6,68 \mathrm{~K}$. Nos résultats montrent explicitement que des dispositifs microponts opérant efficacement d'une part loin de leur température critique et d'autre part dans une gamme de température importante peuvent être réalisés. L'influence de l'harmonique 15 est encore sensible. La largeur des marches ne dépasse pas $60 \mu \mathrm{A}$, ce qui peut résulter de l'effet de shunt de la résistance du canal signalé plus haut. L'hystérésis de la caractéristique $(I, V)$ de la figure 7 est importante par suite de la valeur élevée de $T_{\mathrm{c}}-T \sim 2,5 \mathrm{~K}$, et ne permet pas d'observer l'influence du fondamental et des premiers harmoniques à $4,2 \mathrm{~K}$; cette difficulté doit être facilement surmontée par un choix judicieux de la durée d'usinage.

\subsection{INFLUENCE D'UN CHAMP MAGNÉTIQUE CONTINU.} - Les microponts de niobium sont relativement insensibles à l'action d'un champ magnétique continu, en comparaison des ponts en Sn. Pour ceux-ci, un champ de quelques dixièmes de gauss à 1 gauss suffit pour annuler $I_{\mathrm{c}}$ [6], [7], [9]. La comparaison des figures 7 et 8 montre la modification de la caractéristique $(I, V)$

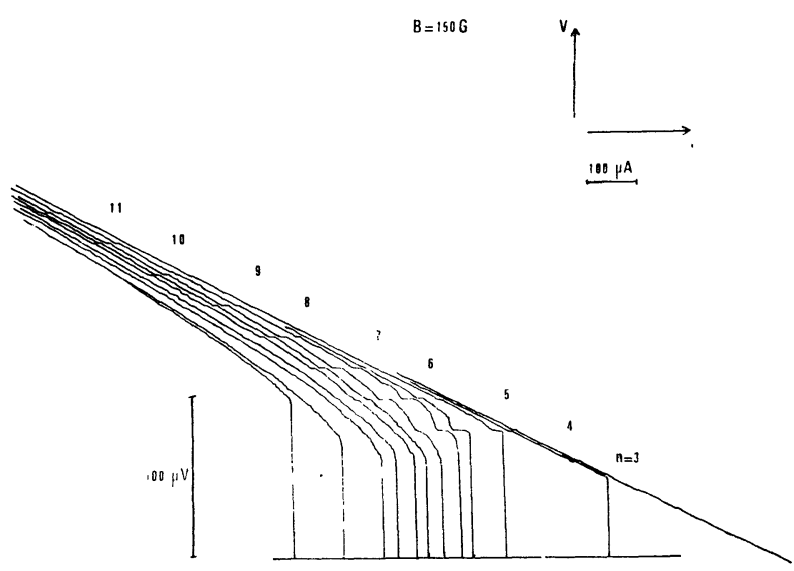

Fig. 8. - Caractéristique $I-V$ à $4,2 \mathrm{~K}$ dans un champ magnétique continu de $150 \mathrm{G}$ perpendiculaire au film supraconducteur du micropont correspondant à la figure 1 , avec pour paramètre la puissance hyperfréquence incidente. 
d'un pont de $\mathrm{Nb}$ pour $B=0$ et $B=150 \mathrm{G}$. Non seulement le courant critique reste pratiquement le même, mais les paliers induits par le rayonnement hyperfréquence à $9000 \mathrm{MHz}$ sont à peine modifiés. Dans la pratique, ceci veut dire que les blindages magnétiques sont inutiles dans les expériences utilisant ce type de micropont.

Les premiers résultats de cette étude indiquent donc en conclusion que la méthode d'usinage par bombar- dement ionique pour la réalisation de microponts supraconducteurs «durs» $(\mathrm{Nb})$ permet de réaliser des dispositifs présentant au moins un certain nombre des qualités que l'on peut exiger.

Remerciements. - Les auteurs remercient M. D. Bouchon de son assistance technique dans les expériences de bombardement ionique et de tests des microponts.

\section{Bibliographie}

[1] Josephson, B. D., Phys. Lett. A 1 (1962) 251 ; Adv. Phys. 14 (1965) 419.

[2] Matisoo, J., Proceedings of the 1972 Applied Superconductivity Conference (Annapolis, Md), IEEE Pub. $n^{\circ} 72$ CHO682-5-TABSC, 535.

[3] Zimmerman, J. E., Applied Supercond. Conf., 1972, 544.

[4] Dayem, A. H. et Wiegand, J. I., Phys. Rev. 155 (1967) 419.

[5] McCumber, D. E., J. Appl. Phys. 39 (1968) 3113.

[6] Gregers-Hansen, P. E., Levinsen, M. T. et Pedersen, G. F., J. Low. Temp. Phys. 7 (1972) 99.

[7] Yeong-Du-Song et Rochlin, G. I., Phys. Rev. Lett. 29 (1972) 416.

[8] Notarys, H. A. et Mercereau, J. E., Physica 55 (1971) 424 (Proc. 1969 Conf. of Superconductivity, Stanford).

[9] Kirschman, R. K., Notarys, H. A. et Mercereau, J. E., Phys. Lett. 34A (1971) 209.
[10] Notarys, H. A., Wang, R. H. et Mercereau, J. E., Proc. of the IEEE 61 (1972) 79.

[11] Castaing, R. et Bernheim, M., 2nd Int. Conf. on Electron and Ion Beam Science and Technology (R. Bakish ed., Gordon and Breach) 1966.

[12] Bernheim, M. et Castaing, R., 4th Int. Conf. on Electron and Ion Beam Science and Technology (Los Angeles, Bakish ed. Gordon and Breach) 1970, 553.

[13] Bernheim, M., Thèse de Docteur Ingénieur, Orsay (1967).

[14] Baratoff, A., Blackburn, J. A. et Schwartz, B. B., Phys. Rev. Lett. 25 (1970) 1096.

[15] Rieger, T. J., Scalapino, D. J., Mercereau, J. E., Phys. Rev. B 6 (1972) 1734.

[16] Cartier, G. et Collingon, J. S., Ion Bombardment of Solids (Heinemann Educational Books Ltd, London). 